Cite this: Nanoscale, 2013, 5, 8884

Received 14th June 2013

Accepted 17th July 2013

DOI: $10.1039 / c 3 n r 03064 d$

\section{Lipid-insertion enables targeting functionalization of erythrocyte membrane-cloaked nanoparticles $\uparrow$}

\author{
Ronnie H. Fang, ${ }^{\text {ab }}$ Che-Ming J. Hu, ${ }^{\text {ab }}$ Kevin N. H. Chen, ${ }^{a}$ Brian T. Luk, ${ }^{\text {bc }}$ \\ Cody W. Carpenter, ${ }^{a}$ Weiwei Gao, ${ }^{\text {ab }}$ Shulin Li, ${ }^{d}$ Dong-Er Zhang, ${ }^{b}$ Weiyue $\mathrm{Lu}^{\mathrm{e}}$ \\ and Liangfang Zhang*ab
}

www.rsc.org/nanoscale

RBC membrane-cloaked polymeric nanoparticles represent an emerging nanocarrier platform with extended circulation in vivo. A lipid-insertion method is employed to functionalize these nanoparticles without the need for direct chemical conjugation. Insertion of both folate and the nucleolin-targeting aptamer AS1411 shows receptor-specific targeting against model cancer cell lines.

A major goal in engineering nanocarriers for systemic drug delivery is to achieve long circulation half-lives, ${ }^{1}$ as increased residence time in the bloodstream can improve the pharmacokinetic profile of therapeutic cargoes and allow a greater chance for the nanocarriers to reach the desired location through either passive ${ }^{2,3}$ or active ${ }^{4-6}$ targeting mechanisms. Ongoing search for new and effective ways to construct longcirculating nanoparticles has introduced numerous stealth functionalization strategies. While the use of synthetic polymers represents the current golden standard for prolonging particle residence time in circulation, recent efforts have drawn inspiration from nature to bestow immune-evasive properties on nanoparticles. ${ }^{7,8}$ Bridging the complex surface biochemistry of nature's carriers, RBCs, with the versatile cargo-carrying capacity of polymeric nanoparticles, an RBC membrane-cloaked nanoparticle (RBC-NP) platform represents a new class of bioinspired nanocarriers with long-circulating capability. ${ }^{9,10}$ In this system, a top-down approach is used to coat polymeric

aDepartment of NanoEngineering, University of California, San Diego, 9500 Gilman Drive, La Jolla, CA 92093, USA. E-mail: zhang@ucsd.edu; Tel: +1-858-246-0999

${ }^{b}$ Moores Cancer Center, University of California, San Diego, 9500 Gilman Drive, La Jolla, CA 92093, USA

'Department of Bioengineering, University of California, San Diego, 9500 Gilman Drive, La Jolla, CA 92093, USA

${ }^{d}$ Department of Pediatric Research, MD Anderson Cancer Center, Houston, TX 77030, USA

${ }^{e}$ Key Laboratory of Smart Drug Delivery, Ministry of Education \& PLA, School of Pharmacy, Fudan University, Shanghai 201203, China

$\dagger$ Electronic supplementary information (ESI) available. See DOI: 10.1039/c3nr03064d nanoparticles with natural RBC membranes, which possess a litany of immunomodulatory proteins responsible for RBCs' extraordinarily long survival in circulation. ${ }^{11-14}$ This membrane cloak acts to camouflage the nanoparticle in vivo to evade immune attacks. ${ }^{9}$ Consisting entirely of biocompatible and biodegradable materials, the RBC-NPs have significant potential for drug delivery applications.

In applying RBC-NPs for disease treatments, particularly against cancers, target-selectivity is a desirable feature that promises minimization of off-target side effects. ${ }^{15-19}$ Cancer targeting has been made possible by carrier functionalization with ligands that target overexpressed tumor antigens, and numerous chemical conjugation techniques employing carboxyl-, amine-, or sulfhydryl-based chemistry have been used to decorate synthetic nanocarriers with targeting ligands. ${ }^{20-26} \mathrm{In}$ the case of biologically derived carriers such as RBC-NPs, however, the presence of biological components on the particle surfaces demands a non-disruptive functionalization strategy since the immune evasion capabilities of cellular membranes are predicated upon having fully functional proteins. In order to address the issue of chemical denaturation, we report a lipidinsertion approach for functionalization of RBC-NPs that exploits the fluidity of bilayered RBC lipid membranes and precludes RBC membrane exposure to chemical reactions. As shown in Fig. 1, targeting moieties are incorporated onto RBC

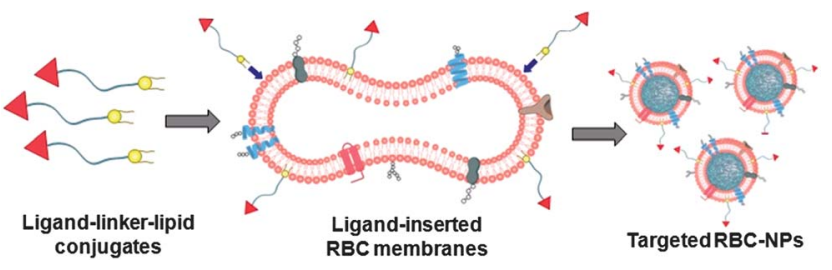

Fig. 1 Schematic of the preparation of RBC-NPs with targeting ability. Ligandlinker-lipid conjugates are synthesized and then inserted into RBC membrane ghosts. The resulting ligand-functionalized RBC membranes are used to coat polymeric cores to form targeted RBC-NPs. 
membranes through the aid of lipid tethers. The physical insertion of ligand-linker-lipid conjugates into the RBC membranes produces functionalized RBC membranes without damaging the existing surface proteins. Using two differently sized ligands, a small molecule folate ( $\mathrm{MW} \sim 441 \mathrm{Da})$ and a nucleolin-targeting aptamer AS1411 (MW $~ 9000$ Da), we demonstrate that the lipid-insertion technique can be applied to targeting ligands of different length scales. Following preparation of RBC-NPs with the functionalized RBC membranes, their receptor-specific targeting ability is verified in model cancer cell lines in vitro.

To demonstrate that lipid-tethered ligands can be spontaneously incorporated onto RBC membranes, a FITC-linker-lipid (excitation/emission $=495 / 519 \mathrm{~nm}$ ) conjugate was used for a proof-of-concept test, where FITC was linked to a lipid molecule through a short polyethylene glycol chain (MW $\sim 2000 \mathrm{Da})$. In the study, $40 \mu \mathrm{g}$ of FITC-PEG-lipid was first incubated with emptied red blood cells (RBC ghosts) collected from $1 \mathrm{~mL}$ of whole mouse blood for $30 \mathrm{~min}$. The RBC ghosts were then centrifuged, washed with PBS, reconstituted, and examined using flow cytometric analysis (Fig. 2A). Compared to unmodified RBC ghosts, modified membrane ghosts had a significantly higher signal under the FITC channel. Visualization by fluorescence microscopy further confirmed the localization of the lipid-tethered FITC on the RBC membranes, as the microscopy image displayed strong FITC
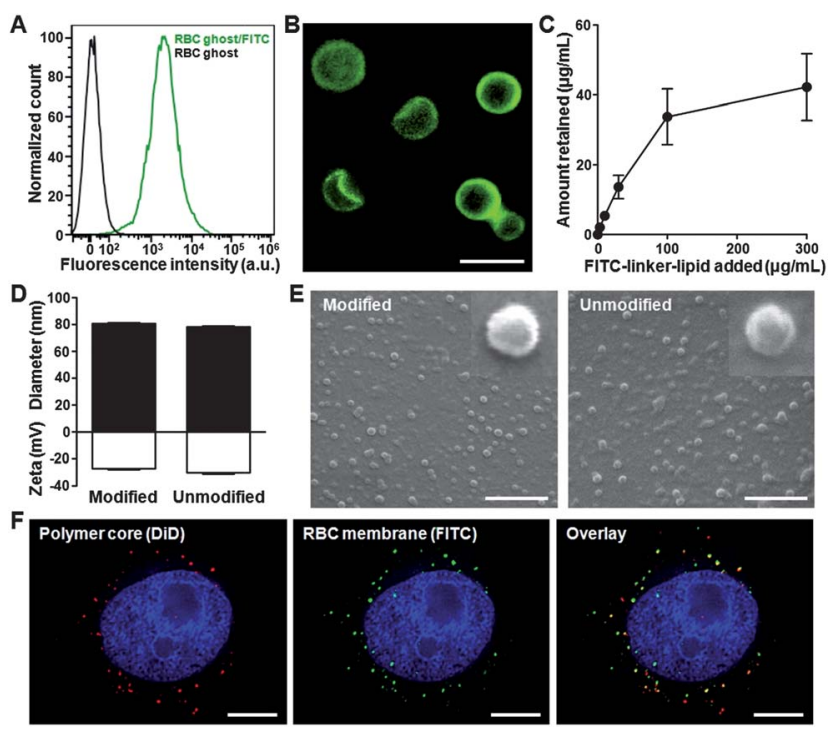

Fig. 2 Lipid-insertion enables modification of RBC-NPs with FITC. (A) Flow cytometry histograms of plain RBC ghosts (black) and RBC ghosts incorporated with FITC-linker-lipid (green). (B) Fluorescence microscopy visualization of RBC ghosts modified with FITC (green). Scale bar $=8 \mu \mathrm{m}$. (C) FITC-linker-lipid was incubated with RBC ghosts derived from $1 \mathrm{~mL}$ of mouse blood. The amount of FITC-linker-lipid incorporated onto the RBC ghosts was then quantified after 30 min of incubation and plotted against the initial input. (D) Physicochemical characterizations (size and zeta potential) of both FITC-modified and unmodified RBC-NPS. (E) SEM images of FITC-modified and unmodified RBC-NPS. Insets represent a single particle with a size of $\sim 80 \mathrm{~nm}$. Scale bars $=500 \mathrm{~nm}$. (F) Colocalization of the polymeric core (red) and the FITC-modified RBC membrane shell (green) upon intracellular uptake by KB cells. Cellular nuclei were stained with DAPI (blue). Scale bars $=8 \mu \mathrm{m}$. signals outlining the exterior of the RBC ghosts (Fig. 2B). To characterize the lipid-insertion efficiency and saturation level, varying amounts of FITC-PEG-lipid were incubated with the membrane ghosts followed by membrane purification through centrifugation. Fluorescence quantification showed that the retained FITC fluorescence increased with the initial FITC-PEGlipid input (Fig. 2C). Saturation was observed as the retained FITC-PEG-lipid approached a plateau at approximately $40 \mu \mathrm{g}$ per $\mathrm{mL}$ of RBCs. Based on the RBC concentration and the molecular weight of the ligand, it is estimated that each RBC ghost contains about 800000 FITC-PEG-lipid. Fig. 2C also demonstrates the adjustability of ligand density on the membranes by controlling the lipid-tethered ligand input.

Upon confirming the incorporation of FITC onto the RBC ghosts, the membrane materials were then used to prepare FITC-modified RBC-NPs. Following a previously reported protocol, ${ }^{9}$ the FITC-modified RBC membrane ghosts were extruded to form $\sim 100 \mathrm{~nm}$ vesicles, which were then mixed and extruded with $70 \mathrm{~nm}$ PLGA particles to generate FITC-modified RBC-NPs. For comparison, RBC-NPs coated with unmodified RBC membranes were also prepared. Characterization of the two particles by dynamic light scattering (DLS) showed similar physicochemical properties between the FITC-modified and unmodified RBC-NPs (Fig. 2D). Both particles were approximately $80 \mathrm{~nm}$ in mean diameter and with a zeta potential below -25 mV. Scanning electron microscopy (SEM) further demonstrated the similarity between the two particle types (Fig. 2E), both of which were spherical in morphology and exhibited monodisperse population distributions. To confirm the colocalization of lipid-tethered ligands with the polymeric cores, an in vitro fluorescence colocalization study was conducted by loading DiD dye (excitation/emission $=644 / 663 \mathrm{~nm}$ ) into the polymeric cores. Following cellular uptake, significant overlap was observed between the DiD-specific red punctates and the FITC-specific green punctates (Fig. 2F). The colocalization pattern confirms the presence of lipid-tethered FITC on the surface of the polymeric cores, demonstrating successful preparation of ligand-modified RBC-NPs.

After validating the lipid-insertion method for RBC-NP functionalization using lipid-tethered fluorescent probes, particle modification with cancer-targeting ligands was explored. A small molecule ligand, folate (MW $~ 441 \mathrm{Da})$, which has a similar molecular weight to FITC, was first examined. Folate-functionalized nanocarriers have broad applicability as folate receptors are overexpressed on several types of cancers. ${ }^{27}$ Upon receptor-mediated binding, folate-functionalized nanocarriers can deliver their cargoes intracellularly through an endocytic uptake pathway. The benefit of folate-induced cancer targeting has been demonstrated on several nanocarrier platforms, ${ }^{28,29}$ and thus its incorporation onto RBC-NPs would improve the particles' utility in cancer drug delivery.

To prepare folate-functionalized RBC-NPs, a commercially available folate-PEG-lipid conjugate was used (Fig. 3A). Folatefunctionalized RBC-NPs were prepared using RBC ghosts inserted with folate-PEG-lipid. Since the targeting ability of folate-functionalized nanoparticles has already been wellestablished, the $\mathrm{KB}$ cell line, a model cancer cell line 
A
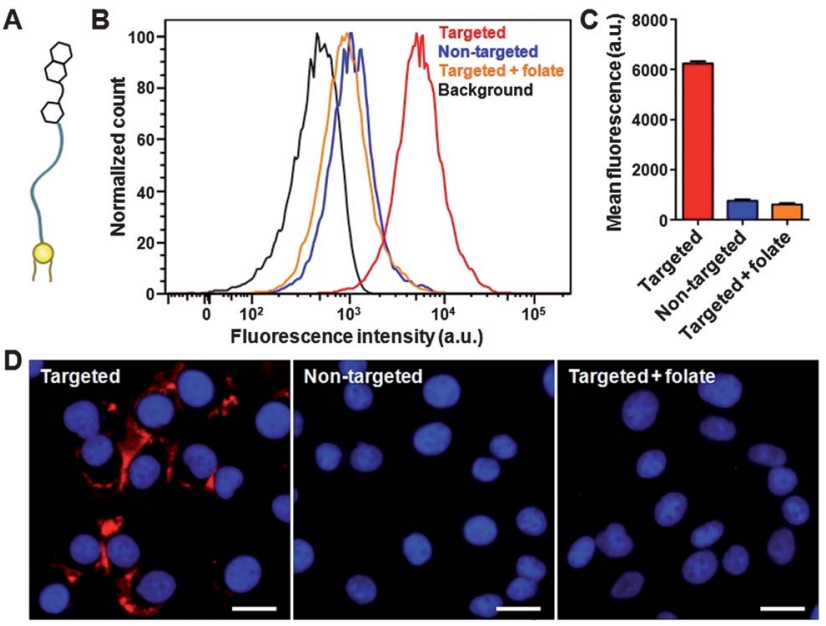

Fig. 3 Lipid-insertion enables targeting functionalization of RBC-NPs with folate (A) Schematic representation of folate-linker-lipid. (B) Flow cytometry histograms of $\mathrm{KB}$ cells alone (black) and the cells incubated with folate-functionalized RBCNPs (red), non-targeted RBC-NPs (blue), and folate-functionalized RBC-NPS together with free folate (orange). (C) Quantification of the mean fluorescence intensity of the histograms in (B). (D) Fluorescence microscopy image of KB cells incubated with folate-functionalized RBC-NPs, non-targeted RBC-NPs, and folatefunctionalized RBC-NPs together with free folate. A fluorescent probe DiD was loaded inside the RBC-NPs for visualization (red) and cellular nuclei were stained with DAPI (blue). Scale bars $=25 \mu \mathrm{m}$.

overexpressing the folate receptor that is commonly used to evaluate folate targeting, ${ }^{30-32}$ was used to confirm successful functionalization of the RBC-NPs. To assess for folate-mediated differential uptake, the cells were cultured in folate-free media and incubated with folate-functionalized RBC-NPs, non-targeted RBC-NPs, or folate-functionalized RBC-NPs together with $1 \mathrm{mM}$ of free folate. The cells from each sample were then detached, washed, and analyzed using flow cytometry (Fig. 3B and C). Compared to the non-targeted RBC-NPs, incubation of folate-functionalized RBC-NPs resulted in an 8-fold increase in cellular uptake. Conjoint incubation with folate-functionalized RBC-NPs and $1 \mathrm{mM}$ of free folate yielded a similar level of cellular uptake as compared to non-targeted RBC-NPs, which indicated that the increased uptake of folate-functionalized RBC-NPs was receptor-specific. Fluorescence microscopy visualization of particle uptake further confirmed the results observed from flow cytometry. As shown in Fig. 3D, fluorescence from the DiD dye encapsulated inside the particles was only observed in cells incubated with folate-functionalized RBC-NPS in the absence of free folate molecules. To demonstrate that the targeting effects were exclusive to cells overexpressing the folate receptor, a negative cancer cell line, A549, ${ }^{33}$ that does not overexpress the folate receptor was incubated with either unmodified or folate-functionalized RBC-NPs. No increased uptake was observed for the targeted nanoparticles compared to the unmodified nanoparticles using both flow cytometry and fluorescence imaging (ESI Fig. S1 $\dagger$ ). It is also important to note that no cytotoxicity was observed for the RBC-NPs when incubated with human umbilical vein endothelial cells (HUVECs), a normal cell line, at the concentrations used in these studies
(ESI Fig. S2 $\dagger$ ). Overall, the results confirm the receptor-specific targeting capability of folate-functionalized RBC-NPs.

To demonstrate that the lipid-insertion method can be applied to targeting ligands of different length scales, particle functionalization with a nucleolin-targeting oligonucleotide, AS1411 aptamer (MW $9000 \mathrm{Da})$, was also tested. Oligonucleotide-based targeting agents, or aptamers, are a versatile class of ligands that can be customized against specific receptors through affinity screening. ${ }^{34}$ AS1411, a 26-mer DNA aptamer with the sequence GGT GGT GGT GGT TGT GGT GGT GGT GG, has shown targeting capability against several cancer cell types owing to frequent overexpression of surface nucleolin on cancerous cells. ${ }^{35-37}$ The ligand has also been applied for the preparation of cancer-targeted nanoparticles, ${ }^{\mathbf{3 8 , 3 9}}$ thus its integration onto the RBC-NPs would greatly benefit the utility of the platform.

To incorporate aptamers onto RBC-NPs via lipid-insertion, a lipid-tethered AS1411 was first prepared. AS1411 aptamers containing a $3^{\prime}$ thiol modifier was reduced using tris[2-carboxyethyl]phosphine (TCEP) and conjugated to lipidPEG-maleimide via maleimide-sulfhydryl chemistry (Fig. 4A). After purification, the AS1411-PEG-lipid conjugates were used to prepare RBC-NPs following the aforementioned procedures. In a cellular uptake study using a surface nucleolin expressing breast cancer cell line, MCF- $7,{ }^{35}$ differential targeting was observed. Flow cytometry analysis revealed that the targeted nanoparticles induced a two-fold increase in cellular uptake as compared to the non-targeted RBC-NPs (Fig. 4B and C). The uptake enhancement by the targeted nanoparticles was also confirmed to be receptor-specific, as blocking by free AS1411

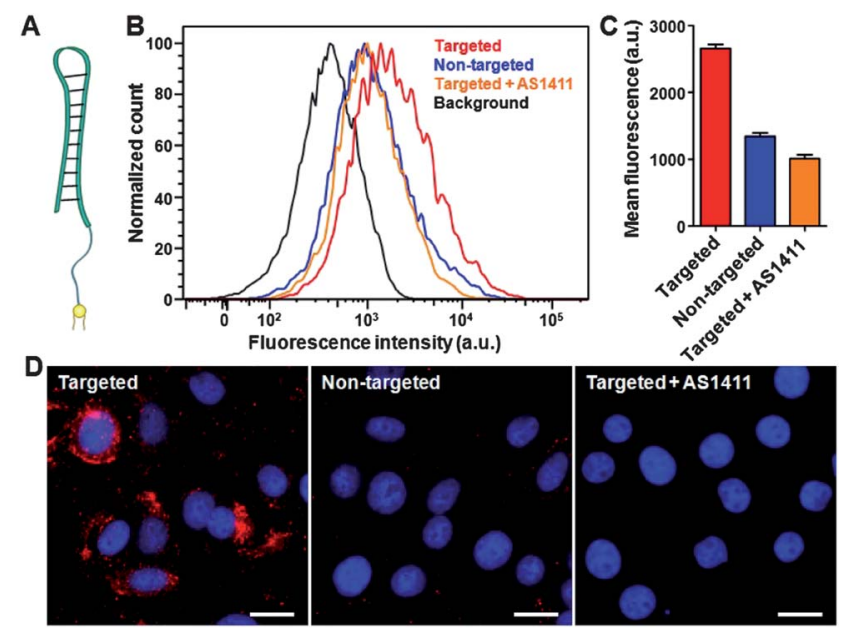

Fig. 4 Lipid-insertion enables targeting functionalization of RBC-NPs with AS1411 aptamer. (A) Schematic representation of AS1411-linker-lipid. (B) Flow cytometry histograms of MCF-7 cells alone (black) and the cells incubated with AS1411-functionalized RBC-NPs (red), non-targeted RBC-NPs (blue), and AS1411functionalized RBC-NPs together with the free AS1411 aptamer (orange). (C) Quantification of the mean fluorescence intensity of the histograms in (B). (D) Fluorescence microscopy of MCF-7 cells incubated with AS1411-functionalized RBC-NPs, non-targeted RBC-NPs, and AS1411-functionalized RBC-NPs together with free AS1411. A fluorescent probe DiD was loaded inside the RBC-NPs for visualization (red) and cellular nuclei were stained with DAPI (blue). Scale bars $=$ $25 \mu \mathrm{m}$. 
reduced the particle uptake to the level of the non-targeted nanoparticles. Fluorescence imaging corroborated the flow cytometry results with the AS1411-fuctionalized RBC-NPs showing much greater uptake than the non-targeted and blocked samples (Fig. 4D). The results demonstrate that the lipid-insertion method can be applied to relatively large targeting ligands (e.g., MW $~ 9000 \mathrm{Da}$ ) with a molecular weight larger than that of the lipid anchor (MW $\sim 748 \mathrm{Da}$ ).

\section{Conclusions}

In summary, by employing a lipid-insertion technique for the functionalization of biological membranes, targeted RBC-NPs were successfully prepared with two different types of targeting ligands. Through the aid of lipid tethers and the dynamic conformation of membrane bilayers, targeting ligands can be spontaneously incorporated onto the RBC-NP platform without exposing the biological membranes to chemical reactions. The robustness and simplicity of this functionalization scheme can enable a wide array of functionalized RBC-NPs for specific disease treatments. In addition, the technique can be generalized to help improve the applicability of emerging biologically inspired nanocarriers possessing complex surface chemistry. The capability to control and adjust ligand density through the lipid-insertion technique also provides versatility for platform optimization. Future studies are warranted to examine the in vivo implications of ligand functionalization on RBC-NPs. The targeted RBC-NPs reported in the present work possess significant potential for cancer treatments as they integrate nature's immune-evasive moieties with cancer-binding ligands.

\section{Acknowledgements}

This work is supported by the National Science Foundation Grant DMR-1216461. R.F. is supported by the Department of Defense (DoD) through the National Defense Science \& Engineering Graduate Fellowship (NDSEG) Program. B.L. is supported by a National Institutes of Health R25CA153915 training grant from the National Cancer Institute.

\section{Notes and references}

1 S. D. Li and L. Huang, Mol. Pharmaceutics, 2008, 5, 496-504.

2 H. Maeda, J. Wu, T. Sawa, Y. Matsumura and K. Hori, J. Controlled Release, 2000, 65, 271-284.

3 A. K. Iyer, G. Khaled, J. Fang and H. Maeda, Drug Discovery Today, 2006, 11, 812-818.

4 R. Singh and J. W. Lillard, Jr, Exp. Mol. Pathol., 2009, 86, 215223.

5 D. F. Emerich and C. G. Thanos, J. Drug Targeting, 2007, 15, 163-183.

6 L. Brannon-Peppas and J. O. Blanchette, Adv. Drug Delivery Rev., 2004, 56, 1649-1659.

7 R. H. Fang, C. M. Hu and L. Zhang, Expert Opin. Biol. Ther., 2012, 12, 385-389.

8 C. M. Hu, R. H. Fang and L. Zhang, Adv. Healthcare Mater., 2012, 1, 537-547.
9 C. M. Hu, L. Zhang, S. Aryal, C. Cheung, R. H. Fang and L. Zhang, Proc. Natl. Acad. Sci. U. S. A., 2011, 108, 1098010985.

10 C. M. Hu, R. H. Fang, J. Copp, B. T. Luk and L. Zhang, Nat. Nanotechnol., 2013, 8, 336-340.

11 D. D. Kim and W. C. Song, Clin. Immunol., 2006, 118, 127136.

12 P. A. Oldenborg, A. Zheleznyak, Y. F. Fang, C. F. Lagenaur, H. D. Gresham and F. P. Lindberg, Science, 2000, 288, 2051-2054.

13 R. K. Tsai and D. E. Discher, J. Cell Biol., 2008, 180, 989-1003.

14 R. K. Tsai, P. L. Rodriguez and D. E. Discher, Blood Cells, Mol., Dis., 2010, 45, 67-74.

15 O. C. Farokhzad and R. Langer, Adv. Drug Delivery Rev., 2006, 58, 1456-1459.

16 M. E. Davis, Z. Chen and D. M. Shin, Nat. Rev. Drug Discovery, 2008, 7, 771-782.

17 D. Peer, J. M. Karp, S. Hong, O. C. Farokhzad, R. Margalit and R. Langer, Nat. Nanotechnol., 2007, 2, 751-760.

18 V. Wagner, A. Dullaart, A. K. Bock and A. Zweck, Nat. Biotechnol., 2006, 24, 1211-1217.

19 L. Zhang, F. X. Gu, J. M. Chan, A. Z. Wang, R. S. Langer and O. C. Farokhzad, Clin. Pharmacol. Ther., 2008, 83, 761-769.

20 K. C. R. Bahadur, B. Thapa and P. S. Xu, Mol. Pharmaceutics, 2012, 9, 2719-2729.

21 D. Bartczak and A. G. Kanaras, Langmuir, 2011, 27, 1011910123.

22 T. Chen, I. Ocsoy, Q. Yuan, R. W. Wang, M. X. You, Z. L. Zhao, E. Q. Song, X. B. Zhang and W. H. Tan, J. Am. Chem. Soc., 2012, 134, 13164-13167.

23 C. M. Hu, S. Kaushal, H. S. T. Cao, S. Aryal, M. Sartor, S. Esener, M. Bouvet and L. Zhang, Mol. Pharmaceutics, 2010, 7, 914-920.

24 E. L. Jin, B. Zhang, X. R. Sun, Z. X. Zhou, X. P. Ma, Q. H. Sun, J. B. Tang, Y. Q. Shen, E. Van Kirk, W. J. Murdoch and M. Radosz, J. Am. Chem. Soc., 2013, 135, 933-940.

25 M. M. J. Kamphuis, A. P. R. Johnston, G. K. Such, H. H. Dam, R. A. Evans, A. M. Scott, E. C. Nice, J. K. Heath and F. Caruso, J. Am. Chem. Soc., 2010, 132, 15881-15883.

26 J. Wang, S. M. Tian, R. A. Petros, M. E. Napier and J. M. DeSimone, J. Am. Chem. Soc., 2010, 132, 11306-11313.

27 J. Sudimack and R. J. Lee, Adv. Drug Delivery Rev., 2000, 41, 147-162.

28 X. Pan and R. J. Lee, Expert Opin. Drug Delivery, 2004, 1, 7-17. 29 J. D. Byrne, T. Betancourt and L. Brannon-Peppas, Adv. Drug Delivery Rev., 2008, 60, 1615-1626.

30 R. J. Lee and P. S. Low, J. Biol. Chem., 1994, 269, 3198-3204.

31 R. J. Lee and P. S. Low, Biochim. Biophys. Acta, Biomembr., 1995, 1233, 134-144.

32 S. Wang, R. J. Lee, G. Cauchon, D. G. Gorenstein and P. S. Low, Proc. Natl. Acad. Sci. U. S. A., 1995, 92, 3318-3322.

33 H. S. Yoo and T. G. Park, J. Controlled Release, 2004, 100, 247256.

34 G. Mayer, M. S. L. Ahmed, A. Dolf, E. Endl, P. A. Knolle and M. Famulok, Nat. Protoc., 2010, 5, 1993-2004.

35 S. Soundararajan, W. W. Chen, E. K. Spicer, N. CourtenayLuck and D. J. Fernandes, Cancer Res., 2008, 68, 2358-2365. 
36 F. Mongelard and P. Bouvet, Curr. Opin. Mol. Ther., 2010, 12, 107-114.

37 S. Soundararajan, L. Wang, V. Sridharan, W. W. Chen, N. Courtenay-Luck, D. Jones, E. K. Spicer and D. J. Fernandes, Mol. Pharmaceutics, 2009, 76, 984-991.
38 A. Aravind, P. Jeyamohan, R. Nair, S. Veeranarayanan, Y. Nagaoka, Y. Yoshida, T. Maekawa and D. S. Kumar, Biotechnol. Bioeng., 2012, 109, 2920-2931.

39 D. W. Hwang, H. Y. Ko, J. H. Lee, H. Kang, S. H. Ryu, I. C. Song, D. S. Lee and S. Kim, J. Nucl. Med., 2010, 51, 98-105. 